Kaolin zeigte nur in einem sehr geringen Teil der Gesamtmasse Färbung durch alle Farbstoffe, die Hauptmasse blieb von allen angewandten Farben unbeeinflußt.

Später hat auch schon Rohland ${ }^{3}$ ) auf die Fähigkeit von Tonen und Zementen, z. B. lösliches Berlinerblau, Anilinblau, Anilinrot, Malachitgrün, Fluoreszein, Aurin, Karmin zurückzuhalten, hingewiesen.

Im Anschlub hieran sei noch die Gelegenheit für einen anderen kleinen Hinweis benutzt. In Heft 4 Band 4 dieser Zeitschrift schreibt Rohland S. 191: "Auch die eingangs erwähnten Vorgänge - es sind Adsorptionsphänomene - hängen mit den kolloiden Eigenschaften der Tone und tonigen Böden zusammen, worauf ich zuerst hingewiesen habe."

Hier muB ein durch Unkenntnis der Sonderfachliteratur erklärlicher Irrtum vorliegen. Es wird zum Beweis dessen genügen, dab ich aus meiner in dieser Zeitschrift früher erschienenen Abhandlung über die Beziehungen der Kolloidforschung zur Agrikulturchemie einen Satz hier wiederhole ${ }^{4}$ ): „V an Bemmelen hat in drei groß angelegten Abhandlungen die Adsorption der Ackererde, ihre Aufnahmefähigkeit für Alkalien und alkalische Erden usw. im weitesten

8) Zeitschr. f. anorg. Chem. 56, 46 (1907).

4) Koll.-Zeitschr. 3, 201 (1908)
Sinne behandelt, und hierbei besonders in der dritten Arbeit auf die überaus groBe Bedeutung hingewiesen, weiche dafür den Kolloiden des Bodens zuzuerkennen ist." -.- Van B e mmelen's Arbeiten erschienen 1878-1888, soweit es sich hier um sie handelt.

Sollte übrigens der Sinn des erwähnten Satzes Rohla nd's bedeuten, daß er zuerst auf die kolloiden Eigenschaften der Tone und der tonigen Böden hingewiesen habe, so mübte auch dies als Irrtum bezeichnet werden. Denn $\mathrm{Sch}$ loesing der Aeltere hat bereits 1872 und $1874^{5}$ ) über die Zusammensetzung der Tone und Kaoline Mitteilung gemacht, in denen or mit großer Entschiedenheit die Kolloidbestandteile des Tors als wesentlich für dessen Eigenschaften hingestellt, und $u$. a. auch dafür experimentelle Nachweise bringt. $\mathrm{Zu}$ etwa gleicher Zeit kam Hilgard unabhängig von ihm $z u$ annähernd den gleichen Anschauungen ${ }^{6}$ ).

Bezüglich der Durchlässigkeit der Ackerböden für Wasser und des Einflusses des Kolloidtones darauf finden sich endlich auch in Schloesing's Arbeiten mannigfache Hinweise, zumal auf die Wirkung der Koagulation der Tone durch Kalksalze. (1874)

5) Compt. rend. 74, 1408 (1872); 79, 376 u. 473 6) Vgl. Hilgard, Soils (New York 1907).

\title{
Ueber die Wirkung von Gelatine im Sahneneis.
}

\section{Von J. Alexander, New York.}

Vor einiger Zeit ${ }^{1}$ ) habe ich auf einer Versammlung in Pittsburg auseinandergesetzt, dab die sehr kleine Menge Gelatine, welche für die Herstellung von Sahneneis verwendet wird, dazu dient, die Bildung von scharf ausgebildeten Kristallen zu verhindern, und somit praktisch gesprochen als Schutzmittel für das Gefüge dient, indem sie das Erzeugnis zart und angenehm für den Geschmack macht. Ich erwähnte ferner, dab die Hauptbestandteile von Sahneneis Kristalloide sind, d.h. Stoffe, welche Kristalle bilden können, während Gelatine ein sehr charakteristisches Glied der Gruppe der nichtkristallisierenden Stoffe, der Kolloide, ist. Damit nun die Folgerungen einiger Experimente mit Gelatine, die ich weiter unten mitteilen will, klarer zutage treten, werde ich zunächst einen kurzen Blick in diese interessante Klasse der Kolloide tun.
Die wissenschaftliche Untersuchung hat ergeben, dab die Kolloide oder, wie man sie auch nenni, Hydrosole in zwei Klassen oder Gruppen eingeteilt werden können, die sich aus dem Verhalten beim Eintrocknen ableiten. Die erste Gruppe, welche diejenigen Kolloide enthält, die wie Gelatine und Gummiarabikum nach dem Eintrocknen wieder aufgelöst werden kö n ne n, heißt die Gruppe der reversiblen Kolloide oder reversiblen Hydrosole. Die Glieder der zweiten Gruppe sind diejenigen, welche nach dem Eintrocknen nicht wieder aufgelöst werden $\mathrm{können,} \mathrm{wie} z$. B. die reinen kolloiden Metalle, Oxyde usw., und heißen demgemäß irreversible Kolloide oder irreversible Hydrosole.

Ein auffallendes Kennzeichen der reinen irreversiblen Kolloide ist ihre große Unbeständig-

1) Vgl. Koll.-Zeitschr. 4, 86 (1909). 
keit; die Anziehungskräfte zwischen den sie bildenden Teilchen scheinen gerade eben zu überwiegen, so daß eine kleine hinzugebrachte Menge Säure oder Salz ihre unmittelbare Koagulation, Flockung oder Fällung hervorruft. Die umkehrbaren Kolloide dagegen sind ganz und gar nicht empfindlich, sondern halten sich unkoaguliert auch beim Hinzubringen der meisten Stoffe.

Die für den vorliegenden Zweck wichtigste Eigenschaft der umkehrbaren Kolloide ist ihre Fähigkeit, auch bei Anwesenheit von kleinen Mengen davon irreversible Kolloide umkehrbar zu machen. So wird reines kolloides Gold, welches ein derart empiindliches irreversibles Hydrosol dars'ellt, daß zur Koagulierung die Verunreinigungen des destillierten Wassers genügen, durch einen Bruchteil eines Prozents hinzugefügter Gelatine ganz unempfi: dlich, auch gegenüber stark koagulierend wirkenden Stoffen. Umkehrbare Kolloide, welche diese hindernde Wirkung ausüben, heißen Schutzkolloide.

In dem Falle des Sahneneises nun bezweckt die Hinzufügung von Gelatine (welche das wirksamste der Schutzkolloide des Handels ist) die Verhütung der Koagulation des Kaseins, welches ein irreversibles Kolloid ist und weiches das wichtigste Proteid, d. h. stickstoffhaltiger Bestandteil der Milch, darstellt. Und aus demselben Grunde macht die Gelatine das Sahneneis leichter verdaulich und damit der Gesundheit zuträglicher. Es ist wohl bekannt, daB die Milch, die wir alle als eine Flüssigkeit kennen, sofort koaguliert, sobald sie mit dem sauren Magensaft in Berührung kommt. In Gegenwart von Gelatine aber wird das Kasein entweder ganz am Koagulieren gehindert oder aber, wenn es doch koaguliert, sind die Gerinnsel so feinkörnig, daß sie sich beim Verdauungsprozeb leicht auflösen.

Dieser Schluß machte mich zunächst stutzig, bis ich beim Suchen in der medizinischen Literatur fand, da $B$ lange vorher von Aerzten durch praktische Versuche entdeckt worden war, daß Gelatine, Gerstenschleim und andere Schutzkolloide wertvolle Zusätze zur Kuhmilch bei der Ernährung von Säuglingen sind. So hat ein Arzt von umfassender Erfahrung, A. Jacobi, welcher heute als erste Autorität in der offentlichen Medizin New Yorks angesehen wird, in seinem Buche: "The Intestinal Diseases of Infancy and Childhood " folgendes ausgesprochen :
"Ebenso geht daraus hervor, dab die anderen Speisen vollständiger verdaut werden und dah eine kleinere Menge Kot gebildet wird, wenn Gelatine hinzugegeben wird als wenn sie weggelassen wird." Und weiter sagt er: "Es kann daher kein $Z$ weifel darüber bestehen, daß ein Zusatz von Gummiarabikum oder Gelatine zur Kuhmilch von Nutzen ist. Diese Stoffe tragen nicht allein dazu bei, die Teilchen der Kuhmilch zu verkleinern und zu verteilen, sondern sie dienen auch unmittelbar als Nahrungsmittel infolge der besseren Ausnutzung. Dazu sind es ganz einfache Stoffe, die überall und billig erhältlich sind, außerhalb des Bereiches der Patentmedizinen liegen und leicht behandelt werden können - ein einfaches Kochen genügt zur Herstellung einer Lösung."

Henry Koplik, eine andere Autorität auf medizinischem Gebiete, sagt in seinem Buche: "Diseases of Infancy and Childhood" auf Seite 65 folgendes:

„Die Menge Kasein in der Kuhmilch ist nicht nur relativ, sondern auch absolut größer als in der Frauenmilch. In der Kuhmilch bildet das Kasein fünf Sechstel der gesamten Proteide, in der Frauenmilch dagegen zwei Sechstel. Diese Tatsache ist von weittragender Bedeutung. Einfache Verdünnung von Kuhmilch ändert an dem gröheren Verhältnis des Kaseins zu den anderen Proteiden gegenüber dem in der menschlichen Milch gar nichts. Umgekehrt koaguliert Kuhmilch bei Zusatz von Säuren oder Salzen sehr rasch; Frauenmilch, wenn überhaupt, sehr langsam. Daher braucht Kuhmilch im Magen des Kindes nur wenig Säure ir Verdauungssaft, um rasch und in dicken Massen zu koagulieren. Frauenmilch dagegen braucht eine größere Menge Säure im Verdauungssaft und koaguliert in feinen Massen. Diese Verschiedenheiten in der Koagulierungsart spielt eine große Rolle beim Studium der Ernährung von Kindern."

Alles dies wird leicht verständlich an der Hand der folgenden Tabellen, welche die mittlere Zusammensetzung von Kuhmilch und Frauenmilch zeigen.

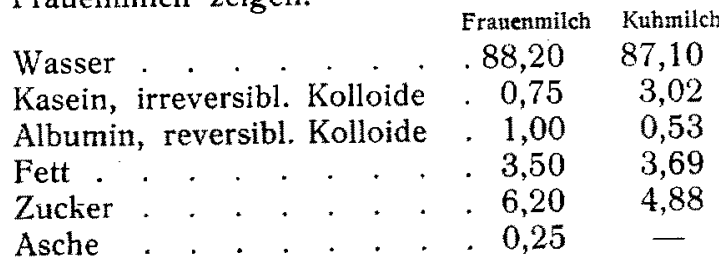

Diese Tatsachen und Angaben werden ferner auch von L. Emmett Holt, einem bekannten 
Kinderspezialisten, in seinem Buche über: "Infancy and Childhood " beglaubigt.

An der Hand eines einfachen Experiments kann man sich von dem Einflusse der Gelatine auf die Koagulation durch Säuren eine Vorstellung machen. Man teilt eine Lösung von Silbernitrat in zwel gleiche Teile und fügt $\mathrm{zu}$ der einen Hälfte ein wenig Gelatinelösung hinzu. Gibt man nun Salzsäure zu jeder Lösung, so beobachtet man in der Lösung ohne Gelatine sofort einen dicken Niederschlag, während die Lösung mit Gelatine nur wolkig erscheint. Beim Filtrieren durch Papierfilter wird der dicke Niederschlag zurückgehalten, woraus hervorgeht, daB seine Teilchen relativ groß sind; die Wolken indessen gehen glatt durchs Filter, zum Beweise, wie fein verteilt die Partikelchen sind.

Die Lösungen von kolloidem Silber und kolloidem Quecksilber verdanken ihre Halt- barkeit und damit ihre ganze Existenz den Schutzkolloiden, welche bei ihrer Herstellung angewendet werden. Nun werden kolloides Silber und Quecksilber in der Medizin in weitem Umfange angewandt und fallen demgemäß unter das Nahrungsmittelgesetz; man hat aber die Produzenten noch nicht aufgefordert, unter Androhung von Strafe den Gebrauch der Kolloide, welche diese Heilmittel erst wirksam machen, zu unterlassen. Wohl aber sind Hersteller von Sahneneis in die ungerechte Lage versetzt worden, daß man von ihnen auf Grund des Nahrungsmittelgesetzes verlangt, ihr Erzeugnis w e n ig e r gesundheitsförderlich herzustellen. Das Nahrungsmittelgesetz ist daran nicht schuld; es liegt vielmehr an der Norm für Sahneneis, welche kein Teil eines Gesetzes sein kann, sondern einfach den Ausdruck der Ansicht derjenigen darstellt, welche es erlassen.

\title{
Einige Beobachtungen an Silberhaloid-Gelen.
}

\author{
Von Dr. Lüp po-Cramer. \\ (Wissenschaftl. Laboratorium der Dr. C. Sch-leuBner-A.-G. zu Frankfurt a. M.) \\ a) $10 \mathrm{ccm}$ Silbernitratlösung 10 Proz., \\ b) dieselbe Silberlösung $+2 \mathrm{ccm}$ einer \\ einprozentigen Lösung von Bleinitrat,
}

In der älteren Literatur über die "Photochromie“, d. h. die Technik zur Herstellung von Photographien in "natürlichen" Farben auf Chlorsilber, findet man mehrfach die Chloride von Blei und Zink als Mittel angegeben, um das im Lichte gefärbte sogenannte Silberchlorür lichtbeständiger $\mathrm{zu}$ machen. Vor allem spielt ein Ueberzug von Bleichlorid und Dextrin, den wohl zuerst $\mathrm{Niepce}$ de St. Victor ${ }^{1}$ ) um 1850 anwandte, zur "Fixierung" jener Bilder eine Rolle.

In der Absicht, meine durch Tageslicht ohne Anwendung eines "Entwicklers" hervorgerufenen latenten Bilder der Röntgenstrahlen, der Radiumstrahlen und der Funkenentladungen ${ }^{2}$ ) für Ausstellungszwecke einigermaBen lichtbeständig zu machen, studierte ich den EinfluB der Bleisalze als Zusatz zu den Gelen der Silberhaloide, wobei einige vom kolloidchemischen Standpunkte vielleicht nicht uninteressante Ergebnisse resultierten.

Setzt man zu einer Lösung von $10 \mathrm{ccm}$ Kaliumbromidlösung 10 Proz. $+10 \mathrm{ccm}$ Wasser

1) W. Zenker, Lehrbuch d. Photochromie (Neuausgabe von B. S c hw a 1 b e, Braunschweig 1900), S. 70.

2) Zusammenfassend beschrieben in meinem soeben erschienenen Buche .Die Röntgenographie* (Halle 1909, bei W. Knapp). so beobachtet man bei kurzem Schütteln der beiden Niederschläge, daß a auf ein kleines Volumen zusammensintert, während $b$ auffallend voluminös bleibt. $a$ ist grünlich weib. b sehr viel heller, fast rein weiß. Der Unterschied in der äuBeren Form der beiden Bromsilberniederschläge bleibt tagelang bestehen, sowohl bei Lichtzutritt, wie im Dunkeln. Im Lichte färbt sich a bald dunkel, während b fast unverändert bleibt. Parallelversuche zeigen, daB das Blei-Bromid, das übrigens in der angegebenen Konzentration in Lösung bleibt, die eigenartige Wirkung ausübt, daß nichi etwa auch Blein it r at ohne UeberschuB von Kaliumbromid über die dem Silbernitrat äquivalente Menge jenen Einfluß auf Gelstruktur und Lichtempfindlichkeit hat. Dieselbe Wirkung beobachtete man auch, wenn man das Bromsilber zuerst $\mathrm{f} u \mathrm{r} \mathrm{s}$ i ch ausfüllt und dann eine. Lösung oder Suspension von reinem Bleibromid zufügt; das bereits auf ein kleines Volumen zusammengesinterte Bromsilber wird bei kurzem Schütteln in Gegenwart des Bleisalzes wieder sehr viel voluminöser. 